\title{
Three solutions to Dirichlet problems for second-order self-adjoint difference equations involving $p$-Laplacian
}

\author{
Feng Xiong ${ }^{1,2}$ and Zhan Zhou ${ }^{1,2^{*}}$ (D)
}

\section{"Correspondence: \\ zzhou0321@hotmail.com 'School of Mathematics and Information Sciences, Guangzhou University, 510006 Guangzhou, P.R. China \\ ${ }^{2}$ Center for Applied Mathematics, Guangzhou University, 510006 \\ Guangzhou, P.R. China}

\section{Springer}

\begin{abstract}
This paper derives several sufficient conditions for the existence of three solutions to the Dirichlet problem for a second-order self-adjoint difference equation involving $p$-Laplacian through the critical point theory. Furthermore, by using the strong maximum principle, we prove that the three solutions are positive under appropriate assumptions on the nonlinearity. Finally, we present three examples to confirm our results.
\end{abstract}

Keywords: Three solutions; $p$-Laplacian; Self-adjoint; Boundary value problem; Critical point theory

\section{Introduction}

For the set of real numbers $\mathbf{R}$, the set of integers $\mathbf{Z}$, and the set of natural numbers $\mathbf{N}$, let us denote $\mathbf{Z}(a)=\{a, a+1, \ldots\}$ and $\mathbf{Z}(a, b)=\{a, a+1, \ldots, b\}$ when $a \leq b$.

Consider the following Dirichlet boundary value problem:

$$
\left\{\begin{array}{l}
\triangle\left[p(k) \phi_{p}(\triangle u(k-1))\right]+\lambda f(k, u(k))=0, \quad k \in \mathbf{Z}(1, T), \\
u(0)=u(T+1)=0
\end{array}\right.
$$

where $T \in \mathbf{N}, \Delta$ is the forward difference operator denoted by $\Delta u(k)=u(k+1)-u(k)$, $\triangle^{2} u(k)=\Delta(\Delta u(k)), \phi_{p}$ is the $p$-Laplacian operator, namely, $\phi_{p}(s)=|s|^{p-2} s, p>1, f(k, \cdot) \in$ $C(\mathbf{R}, \mathbf{R})$ for each $k \in \mathbf{Z}(1, T)$.

Difference equations have been widely applied as mathematical models in biology, physics, and other research fields [1-5]. For instance, the qualitative analysis of equations with $p$-Laplacian-like operators has become an important research topic due to the fact that these equations arise in a variety of real world problems such as in the study of nonNewtonian fluid theory and the turbulent flow of a polytrophic gas in a porous medium; see [6-8] for more details. Accordingly, the research of the difference equations has attracted much attention in recent years. The upper and lower solution techniques, as well as the fixed point methods $[9,10]$, are useful tools in researching the BVPs of difference equations. In 2003, the second-order difference equation was first studied by Yu and Guo

(c) The Author(s) 2021. This article is licensed under a Creative Commons Attribution 4.0 International License, which permits use, sharing, adaptation, distribution and reproduction in any medium or format, as long as you give appropriate credit to the original author(s) and the source, provide a link to the Creative Commons licence, and indicate if changes were made. The images or other third party material in this article are included in the article's Creative Commons licence, unless indicated otherwise in a credit line to the material. If material is not included in the article's Creative Commons licence and your intended use is not permitted by statutory regulation or exceeds the permitted use, you will need to obtain permission directly from the copyright holder. To view a copy of this licence, visit http://creativecommons.org/licenses/by/4.0/. 
[11] via the critical point theory, and some results on the existence of periodic solutions and subharmonic solutions were obtained. Since then, many researchers have explored the difference equations by mainly using the critical point theory to show a lot of interesting results on BVPs [12-19], periodic solutions [11, 20-22], and homoclinic solutions [23-33].

In [21], Yu et al. studied the following second-order difference equation:

$$
\Delta[p(t) \Delta u(t-1)]+q(t) u(t)=f(t, u(t)), \quad t \in \mathbf{Z} .
$$

Based on the critical point theory, some sufficient conditions to prove the existence of periodic solutions of (1.2) were derived. In [31], the variational method was also explored to prove the existence of nontrivial homoclinic orbits for (1.2).

In [34], Ma studied the following homogeneous and linear difference equation:

$$
\Delta[p(t) \Delta u(t-1)]+q(t) u(t)=0, \quad t \in \mathbf{Z}(1)
$$

and some results on recessive and dominant solutions were established. In [35], Long, Yu, and Guo considered the disconjugacy and the C-disfocality of (1.3), and several sufficient conditions were derived explicitly in terms of equation coefficients.

For $p(k) \equiv 1$ in (1.1), Jiang and Zhou [36] showed the existence of three solutions of (1.1). The aim of this paper is to prove that the three solutions exist for (1.1) by using different methods. Moreover, by using a new strong maximum principle established in this paper, we prove that the three solutions are positive under suitable conditions. Even in the special case $p(k) \equiv 1$, the existence results of three nontrivial solutions for (1.1) are new. However, as shown in the last Example 4.3, the conclusion about the three solutions using [36, Theorem 3.1] cannot be acquired.

The rest of this paper is organized as follows. Some preliminaries are presented in Sect. 2. Our main results are given in Sect. 3. Finally, we give three examples to confirm our findings in Sect. 4.

\section{Preliminaries}

Let $X$ denote a finite-dimensional real Banach space and let $I_{\lambda}: X \rightarrow \mathbf{R}$ be a functional satisfying the following structure hypothesis:

$\left(H_{1}\right) I_{\lambda}(u)=\Phi(u)-\lambda \Psi(u)$ for $u \in X$, where $\lambda>0, \Phi, \Psi: X \rightarrow \mathbf{R}$ are two continuous functions of class $C^{1}$ on $X$ with $\Phi$ is coercive, that is, $\lim _{\|u\| \rightarrow \infty} \Phi(u)=+\infty$.

The following lemma comes from Corollary 3.1 of [37].

Lemma 2.1 Assume that $\left(H_{1}\right)$ holds together with the following conditions:

$\left(H_{2}\right) \Phi$ is convex and $\inf _{X} \Phi=\Phi(0)=\Psi(0)=0$;

$\left(H_{3}\right)$ If $x_{1}, x_{2}$ are local minima for the functional $I_{\lambda}(u)=\Phi(u)-\lambda \Psi(u)$ such that $\Psi\left(x_{1}\right) \geq 0$ and $\Psi\left(x_{2}\right) \geq 0$, then

$$
\inf _{t \in[0,1]} \Psi\left(t x_{1}+(1-t) x_{2}\right) \geq 0
$$

Further, assume that there are two positive constants $\rho_{1}, \rho_{2}$ and $\bar{v} \in X$, with $\rho_{1}<\Phi(\bar{v})<\frac{\rho_{2}}{2}$, such that 
(i) $\frac{\sup _{u \in \Phi^{-1}\left(-\infty, \rho_{1}\right)} \Psi(u)}{\rho_{1}}<\frac{1}{2} \frac{\Psi(\bar{v})}{\Phi(\bar{v})}$,

(ii) $\frac{\sup _{u \in \Phi^{-1}\left(-\infty, \rho_{2}\right)} \Psi(u)}{\rho_{2}}<\frac{1}{4} \frac{\Psi(\bar{v})}{\Phi(\bar{v})}$.

Then, for $\lambda \in\left(\frac{2 \Phi(\bar{v})}{\Psi(\bar{v})}, \min \left\{\frac{\rho_{1}}{\sup _{u \in \Phi^{-1}\left(-\infty, \rho_{1}\right)} \Psi(u)}, \frac{\rho_{2} / 2}{\sup _{u \in \Phi^{-1}\left(-\infty, \rho_{2}\right)} \Psi(u)}\right\}\right)$, the functional $I_{\lambda}$ admits at least three distinct critical points $u_{1}, u_{2}, u_{3}$ such that $u_{1} \in \Phi^{-1}\left(-\infty, \rho_{1}\right), u_{2} \in \Phi^{-1}\left(\rho_{1}, \rho_{2} / 2\right)$, and $u_{3} \in \Phi^{-1}\left(-\infty, \rho_{2}\right)$.

Let us introduce another condition as follows:

$(\Lambda)$ Let $(X,\|\cdot\|)$ be a real finite-dimensional Banach space, and let $\Phi, \Psi: X \rightarrow \mathbf{R}$ be two continuously Gâteaux differentiable functionals, with $\Phi$ being coercive and $\inf _{X} \Phi=$ $\Phi(0)=\Psi(0)=0$.

Lemma 2.2 ([38, Theorem 4.1]) Assume that $(\Lambda)$ holds and there exist $r>0$ and $\bar{x} \in X$, with $r<\Phi(\bar{x})$, such that

$\left(a_{1}\right) \frac{\sup _{\Phi(x) \leq r} \Psi(x)}{r}<\frac{\Psi(\bar{x})}{\Phi(\bar{x})}$,

$\left(a_{2}\right)$ For each $\lambda \in \Lambda_{r}:=\left(\frac{\Phi(\bar{x})}{\Psi(\bar{x})}, \frac{r}{\sup _{\Phi(x) \leq r} \Psi(x)}\right)$, the functional $\Phi-\lambda \Psi$ is coercive.

Then, for each $\lambda \in \Lambda_{r}$, the functional $\Phi-\lambda \Psi$ has at least three distinct critical points in $X$.

Consider the $T$-dimensional Banach space

$$
X=\{u: \mathbf{Z}(0, T+1) \rightarrow R: u(0)=u(T+1)=0\}
$$

equipped with a norm $\|\cdot\|$ given by

$$
\|u\|=\left(\sum_{k=1}^{T+1}|\Delta u(k-1)|^{p}\right)^{\frac{1}{p}}, \quad u \in X
$$

Define two functionals on $X$ as follows:

$$
\Phi(u)=\frac{1}{p} \sum_{k=1}^{T+1} p(k)|\Delta u(k-1)|^{p}, \quad \Psi(u)=\sum_{k=1}^{T} F(k, u(k)), \quad u \in X,
$$

where $F(k, \xi)=\int_{0}^{\xi} f(k, s) d s$ for $\xi \in \mathbf{R}$. Obviously, $\Phi, \Psi \in C^{1}(X, R)$.

Define

$$
I_{\lambda}(u)=\Phi(u)-\lambda \Psi(u), \quad u \in X .
$$

Through careful calculations, for each $u, v \in X$, we see that

$$
\begin{aligned}
I_{\lambda}^{\prime}(u)(v) & =\lim _{t \rightarrow 0} \frac{I_{\lambda}(u+t v)-I_{\lambda}(u)}{t} \\
& =\sum_{k=1}^{T+1} p(k)|\Delta u(k-1)|^{p-2} \Delta u(k-1) \Delta v(k-1)-\lambda \sum_{k=1}^{T} f(k, u(k)) v(k) \\
& =\sum_{k=1}^{T} p(k) \phi_{p}(\Delta u(k-1)) \Delta v(k-1)-p(T+1) \phi_{p}(\Delta u(T)) v(T)
\end{aligned}
$$




$$
\begin{aligned}
& -\lambda \sum_{k=1}^{T} f(k, u(k)) v(k) \\
= & \left.p(k) \phi_{p}(\Delta u(k-1)) v(k-1)\right|_{1} ^{T+1}-\sum_{k=1}^{T} \Delta\left[p(k) \phi_{p}(\Delta u(k-1))\right] v(k) \\
& -p(T+1) \phi_{p}(\Delta u(T)) v(T)-\lambda \sum_{k=1}^{T} f(k, u(k)) v(k) \\
= & -\left\{\sum_{k=1}^{T} \Delta\left[p(k) \phi_{p}(\Delta u(k-1))\right]+\lambda \sum_{k=1}^{T} f(k, u(k))\right\} v(k) .
\end{aligned}
$$

Thus

$$
I_{\lambda}^{\prime}(u)(v)=-\sum_{k=1}^{T}\left\{\Delta\left[p(k) \phi_{p}(\Delta u(k-1))\right]+\lambda f(k, u(k))\right\} v(k)=0
$$

is equivalent to

$$
\triangle\left[p(k) \phi_{p}(\Delta u(k-1))\right]+\lambda f(k, u(k))=0
$$

for any $k \in \mathbf{Z}(1, T)$ with $u(0)=u(T+1)=0$. That is, each critical point of the functional $I_{\lambda}$ corresponds to a solution of (1.1). Therefore, we simplify the solution of (1.1) into the problem of finding the critical points of $I_{\lambda}$ on $X$.

Lemma 2.3 ([36]) For any $u \in X$ and $p>1$, one has

$$
\max _{k \in Z(1, T)}|u(k)| \leq \frac{(T+1)^{(p-1) / p}}{2}\|u\|
$$

Lemma 2.4 Assume that $u \in X$ is such that either

$$
u(k)>0 \quad \text { or } \quad-\triangle\left[p(k) \phi_{p}(\triangle u(k-1))\right] \geq 0,
$$

for $k \in Z(1, T)$. Then, either $u(k)>0$ for all $k \in Z(1, T)$ or $u \equiv 0$.

Proof Let $j \in \mathbf{Z}(1, T)$ and

$$
u(j)=\min \{u(k): k \in N(1, T)\}
$$

If $u(j)>0$, then it is clear that $u(k)>0$ for all $k \in \mathbf{Z}(1, T)$. If $u(j) \leq 0$, then $u(j)=\min \{u(k)$ : $k \in N(0, T+1)\}$, since $\Delta u(j-1)=u(j)-u(j-1) \leq 0$ and $\Delta u(j)=u(j+1)-u(j) \geq 0$. As $\phi_{p}(0)=0$ and $\phi_{p}(s)$ is increasing in $s$, we obtain

$$
\phi_{p}(\Delta u(j)) \geq 0 \geq \phi_{p}(\Delta u(j-1)) .
$$

Owing to $p(j+1)>0$ and $p(j)>0$, it holds that

$$
p(j+1) \phi_{p}(\Delta u(j)) \geq 0 \geq p(j) \phi_{p}(\triangle u(j-1)) .
$$


On the other hand, by (2.6), we have

$$
p(j+1) \phi_{p}(\Delta u(j)) \leq p(j) \phi_{p}(\Delta u(j-1))
$$

By combining (2.8) with (2.9), we have $p(j+1) \phi_{p}(\Delta u(j))=0=p(j) \phi_{p}(\Delta u(j-1))$.

Thus, $u(j+1)=u(j-1)=u(j)$. If $j+1=T+1$, then we can get $u(j)=0$. Otherwise, $j+1 \in N(1, T)$. Replacing $j$ by $j+1$, we can get $u(j+2)=u(j+1)$. Continuing this process $T+1-j$ times, we have $u(j)=u(j+1)=u(j+2)=\cdots=u(T+1)=0$. Similarly, we have $u(j)=u(j-1)=u(j-2)=\cdots=u(0)=0$. Thus, $u \equiv 0$ and the proof is completed.

\section{Main results}

For two positive constants $c$ and $q$, we denote

$$
\Gamma(c)=\frac{\sum_{k=1}^{T} F(k, c)}{c^{p}}, \quad F^{q}:=\sum_{k=1}^{T} F(k, q), \quad \min _{k \in \mathbf{Z}(1, T)}\{p(k)\}=m>0 .
$$

Theorem 3.1 Suppose that for $k \in Z(1, T), f(k, u)$ is a continuous function with respect to $u$, and $c_{1}, c_{2}$, and $d$ are positive constants, with

$$
\frac{2 c_{1} m^{\frac{1}{P}}}{(T+1)^{\frac{p-1}{p}}(p(1)+p(T+1))^{\frac{1}{p}}}<d<\frac{2 c_{2} m^{\frac{1}{P}}}{(T+1)^{\frac{p-1}{p}}(2(p(1)+p(T+1)))^{\frac{1}{p}}},
$$

such that

$\left(e_{1}\right) \max \left\{\Gamma\left(c_{1}\right), 2 \Gamma\left(c_{2}\right)\right\}<\frac{2^{p-1} m}{(p(1)+p(T+1))(T+1)^{p-1}} \Gamma(d)$.

Then, for each

$$
\lambda \in\left(\frac{2(p(1)+p(T+1))}{p \Gamma(d)}, \frac{m 2^{p}}{p\left((T+1)^{p-1}\right) \max \left\{\Gamma\left(c_{1}\right), 2 \Gamma\left(c_{2}\right)\right\}}\right),
$$

problem (1.1) has at least three solutions $u^{i}(i=1,2,3)$, with $u^{1} \in \Phi^{-1}\left(-\infty, \rho_{1}\right), u^{2} \in$ $\Phi^{-1}\left(\rho_{1}, \rho_{2} / 2\right)$, and $u^{3} \in \Phi^{-1}\left(-\infty, \rho_{2}\right)$, where $\rho_{1}=\frac{m\left(2 c_{1}\right)^{p}}{p\left((T+1)^{p-1}\right)}, \rho_{2}=\frac{m\left(2 c_{2}\right)^{p}}{p\left((T+1)^{p-1}\right)}$.

Proof Our idea is to use Lemma 2.1 to confirm our conclusion. First, we prove the coercivity of $\Phi$. Since

$$
\begin{aligned}
\Phi(u) & =\frac{1}{p} \sum_{k=1}^{T+1} p(k)|\Delta u(k-1)|^{p} \\
& \geq \frac{1}{p} m \sum_{k=1}^{T+1}|\Delta u(k-1)|^{p} \\
& =\frac{m}{p}\|u\|^{p}
\end{aligned}
$$

we see that $\Phi$ is coercive.

Next, we prove that $\Phi$ is convex. Consider $y=x^{p}$ for $x \geq 0$. By taking the derivative of $y$ with respect to $x$, we have

$$
y^{\prime}=p x^{p-1}, \quad y^{\prime \prime}=p(p-1) x^{p-2} \geq 0
$$


So, $y=x^{p}$ is convex on $[0,+\infty)$. That is, $\frac{x_{1}^{p}+x_{2}^{p}}{2} \geq\left(\frac{x_{1}+x_{2}}{2}\right)^{p}$. From

$$
\begin{aligned}
\Phi\left(\frac{u_{1}+u_{2}}{2}\right) & =\frac{1}{p} \sum_{k=1}^{T} p(k)\left|\triangle \frac{u_{1}(k)+u_{2}(k)}{2}\right|^{p} \\
& \leq \frac{1}{p} \sum_{k=1}^{T} p(k)\left(\frac{\left|\Delta u_{1}(k)\right|+\left|\Delta u_{2}(k)\right|}{2}\right)^{p} \\
& \leq \frac{1}{p} \sum_{k=1}^{T} p(k)\left(\frac{\left|\Delta u_{1}(k)\right|^{p}+\left|\Delta u_{2}(k)\right|^{p}}{2}\right) \\
& =\frac{1}{2}\left(\frac{1}{p} \sum_{k=1}^{T} p(k)\left|\Delta u_{1}(k)\right|^{p}+\frac{1}{p} \sum_{k=1}^{T} p(k)\left|\Delta u_{2}(k)\right|^{p}\right) \\
& =\frac{1}{2}\left(\Phi\left(u_{1}\right)+\Phi\left(u_{2}\right)\right),
\end{aligned}
$$

we know that $\Phi$ is convex. From the definitions of $\Phi$ and $\Psi$, we have

$$
\inf _{X} \Phi=\Phi(0)=\Psi(0)=0
$$

Therefore, $\Phi$ and $\Psi$ satisfy hypotheses $\left(H_{1}\right)$ and $\left(H_{2}\right)$ of Lemma 2.1. Now, let $x_{1}$ and $x_{2}$ be two local minima for $I_{\lambda}$. Then, $x_{1}$ and $x_{2}$ are two critical points of $I_{\lambda}$. In this case, $x_{1}$ and $x_{2}$ are two solutions of (1.1). By Lemma 2.4, we have $x_{1} \geq 0$ and $x_{2} \geq 0$. Consequently, we obtain $t x_{1}+(1-t) x_{2} \geq 0$ for $t \in[0,1]$. It follows that $\Psi\left(t x_{1}+(1-t) x_{2}\right) \geq 0$ and $\left(H_{3}\right)$ is verified.

When $\|u\| \leq\left(\frac{p \rho_{1}}{m}\right)^{\frac{1}{p}}$, by Lemma 2.3, we have

$$
\max _{k \in \mathbf{Z}(1, T)}|u(k)| \leq \frac{(T+1)^{(p-1) / p}}{2}\left(\frac{p \rho_{1}}{m}\right)^{\frac{1}{p}}=c_{1} .
$$

Similarly, it holds that

$$
\max _{k \in \mathbf{Z}(1, T)}|u(k)| \leq \frac{(T+1)^{(p-1) / p}}{2}\left(\frac{p \rho_{2}}{m}\right)^{\frac{1}{p}}=c_{2}
$$

for $u \in X$ with $\|u\| \leq\left(\frac{p \rho_{2}}{m}\right)^{\frac{1}{p}}$. Taken together, we have

$$
\begin{aligned}
\frac{\sup _{u \in \Phi^{-1}\left(-\infty, \rho_{1}\right)} \Psi(u)}{\rho_{1}} & \leq \frac{\sup _{\|u\|<\left(\frac{p \rho_{1}}{m}\right)^{\frac{1}{p}}} \sum_{k=1}^{T} F(k, u(k))}{\rho_{1}} \\
& \leq \frac{\sum_{k=1}^{T} F\left(k, c_{1}\right)}{\rho_{1}} \\
& =\frac{p\left((T+1)^{p-1}\right)}{m\left(2 c_{1}\right)^{p}} \sum_{k=1}^{T} F\left(k, c_{1}\right) \\
& =\frac{p\left((T+1)^{p-1}\right)}{m 2^{p}} \Gamma\left(c_{1}\right),
\end{aligned}
$$


and

$$
\begin{aligned}
\frac{\sup _{u \in \Phi^{-1}\left(-\infty, \rho_{2}\right)} \Psi(u)}{\rho_{2}} & \leq \frac{\sup _{\left.\|u\|<<\frac{p \rho_{2}}{m}\right)^{\frac{1}{p}} \sum_{k=1}^{T} F(k, u(k))}^{\rho_{2}}}{} \\
& \leq \frac{\sum_{k=1}^{T} F\left(k, c_{2}\right)}{\rho_{2}} \\
& =\frac{p\left((T+1)^{p-1}\right)}{m\left(2 c_{2}\right)^{p}} \sum_{k=1}^{T} F\left(k, c_{2}\right) \\
& =\frac{p\left((T+1)^{p-1}\right)}{m 2^{p}} \Gamma\left(c_{2}\right) .
\end{aligned}
$$

By (3.4) and (3.5), we verify assumptions (i) and (ii) of Lemma 2.1.

On the other hand, select $\bar{v} \in X$ such that

$$
\bar{v}= \begin{cases}d, & \text { if } k \in \mathbf{Z}(1, T), \\ 0, & \text { if } k=0 \text { or } k=T+1 .\end{cases}
$$

Then we have $\Phi(\bar{v})=\frac{(p(1)+p(T+1)) d}{p}$. Hence, from (3.1), we obtain

$$
\rho_{1}<\Phi(\bar{v})<\frac{\rho_{2}}{2} .
$$

Moreover, we have

$$
\frac{2 \Phi(\bar{v})}{\Psi(\bar{v})}=\frac{2(p(1)+p(T+1))}{p \Gamma(d)} .
$$

By Lemma 2.1, for $\lambda \in\left(\frac{2(p(1)+p(T+1))}{p \Gamma(d)}, \frac{m 2^{p}}{p\left((T+1)^{p-1}\right) \max \left\{\Gamma\left(c_{1}\right), 2 \Gamma\left(c_{2}\right)\right\}}\right)$, problem (1.1) has at least three solutions $u^{i}(i=1,2,3)$, and

$$
\begin{aligned}
& u^{1} \in \Phi^{-1}\left(-\infty, \frac{m\left(2 c_{1}\right)^{p}}{p\left((T+1)^{p-1}\right)}\right), \\
& u^{2} \in \Phi^{-1}\left(\frac{m\left(2 c_{1}\right)^{p}}{p\left((T+1)^{p-1}\right)}, \frac{m\left(2 c_{2}\right)^{p}}{2 p\left((T+1)^{p-1}\right)}\right), \\
& u^{3} \in \Phi^{-1}\left(-\infty, \frac{m\left(2 c_{2}\right)^{p}}{p\left((T+1)^{p-1}\right)}\right) .
\end{aligned}
$$

Thus, the proof of Theorem 3.1 is completed.

Let

$$
f(k, u)= \begin{cases}\alpha(k) y(u), & \text { if } u \geq 0 \\ 0, & \text { if } u<0\end{cases}
$$

for all $(k, u) \in \mathbf{Z}(1, T) \times \mathbf{R}$. 
Next, we consider the following discrete system:

$$
\left\{\begin{array}{l}
\triangle\left[p(k) \phi_{p}(\Delta u(k-1))\right]+\lambda \alpha(k) y(u(k))=0, \quad k \in \mathbf{Z}(1, T) \\
u(0)=u(T+1)=0
\end{array}\right.
$$

where $\alpha: \mathbf{Z}(1, T) \rightarrow \mathbf{R}$ is a continuous function and $y:[0,+\infty) \rightarrow \mathbf{R}$ is a nonnegative continuous function with $y(0)=0$.

Corollary 3.1 Suppose that $c_{1}, c_{2}$, and d are positive constants satisfying (3.1), and $\left(e_{2}\right) \max \left\{\frac{\int_{0}^{c_{1}} y(\xi) d \xi}{c_{1}^{p}}, \frac{2 \int_{0}^{c_{2}} y(\xi) d \xi}{c_{2}^{p}}\right\}<\frac{m 2^{p-1} \int_{0}^{d} y(\xi) d \xi}{(p(1)+p(T+1))(T+1)^{p-1} d^{p}}$.

Then, for each

$$
\lambda \in \frac{1}{\sum_{k=1}^{T} \alpha(k)}\left(\frac{2(p(1)+p(T+1)) d^{p}}{p \int_{0}^{d} y(\xi) d \xi}, \frac{m 2^{p}}{p\left((T+1)^{p-1}\right) \max \left\{\frac{\int_{0}^{c_{1}} y(\xi) d \xi}{c_{1}^{p}}, \frac{2 \int_{0}^{c_{2}} y(\xi) d \xi}{c_{2}^{p}}\right\}}\right),
$$

problem (3.7) has at least two positive solutions.

Next, let us give another theorem as follows:

Theorem 3.2 Suppose that $c$ and $d$ are positive constants such that

$$
\frac{1}{p}|d|^{p}(p(1)+p(T+1))>\frac{m(2 c)^{p}}{p(T+1)^{p-1}}
$$

and satisfying

(i) $f(k, \xi)>0$ for each $k \in Z(1, T)$ and $\xi \in[-c, d]$;

(ii) $\frac{p F^{d}}{|d|^{p}(p(1)+p(T+1))}>\frac{F^{c} p(T+1)^{p-1}}{m(2 c)^{p}}$;

(iii) $\lim \sup _{|\xi| \rightarrow+\infty} \frac{F(k, \xi)}{|\xi|^{p}}<\frac{F^{c}}{T c^{p}}$.

Then, for each $\lambda \in \Lambda_{r}:=\left(\frac{|d|^{p}(p(1)+p(T+1))}{p F^{d}}, \frac{m(2 c)^{p}}{F^{c} p(T+1)^{p-1}}\right)$, problem (1.1) has at least three nontrivial solutions.

Proof Apparently, condition $(\Lambda)$ in Lemma 2.2 is tenable. Theorem 3.2 can be established after verifying $\left(a_{1}\right)$ and $\left(a_{2}\right)$ in Lemma 2.2 .

Let

$$
r=\frac{m(2 c)^{p}}{p(T+1)^{p-1}}
$$

For $u \in X$ and $\Phi(u) \leq r$, according to (3.2) and due to $\|u\| \geq 1$, we have

$$
\|u\| \leq\left(\frac{p}{m} \Phi(u)\right)^{\frac{1}{p}} \leq\left(\frac{p}{m} r\right)^{\frac{1}{p}}=\frac{2 c}{(T+1)^{\frac{p-1}{p}}}
$$

Using Lemma 2.3, we have $\|u\|_{\infty} \leq c$. Thus,

$$
\begin{aligned}
\frac{\sup _{\Phi(u) \leq r} \Psi(u)}{r} & \leq \frac{\sup _{\|u\|_{\infty} \leq c} \sum_{k=1}^{T} F(k, u(k))}{r} \\
& \leq \frac{\sum_{k=1}^{T} \max _{|s| \leq c} F(k, s)}{r}=\frac{F^{c} p(T+1)^{p-1}}{m(2 c)^{p}} .
\end{aligned}
$$


Now, fix

$$
\bar{u}(k)= \begin{cases}d, & \text { if } k \in \mathbf{Z}(1, T) \\ 0, & \text { if } k=0 \text { or } k=T+1\end{cases}
$$

Clearly, $\bar{u} \in X$. It follows from (3.8) that

$$
\Phi(\bar{u})=\frac{1}{p}|d|^{p}(p(1)+p(T+1))>\frac{m(2 c)^{p}}{p(T+1)^{p-1}}=r .
$$

Moreover, one has

$$
\frac{\Psi(\bar{u})}{\Phi(\bar{u})}=\frac{p \sum_{k=1}^{T} F(k, d)}{|d|^{p}(p(1)+p(T+1))}=\frac{p F^{d}}{|d|^{p}(p(1)+p(T+1))} .
$$

Therefore, by (3.9) and (3.10), condition $\left(a_{1}\right)$ follows.

In order to verify the coercivity of the functional $\Phi-\lambda \Psi$, we first suppose that

$$
\limsup _{|\xi| \rightarrow+\infty} \frac{F(k, \xi)}{|\xi|^{p}} \geq 0
$$

According to condition (iii), there exists $\varepsilon$ such that $\lim \sup _{|\xi| \rightarrow+\infty} \frac{F(k, \xi)}{|\xi|^{p}}<\varepsilon<\frac{F^{c}}{T c^{p}}$. Thus, there exists a positive constant $h_{\varepsilon}$ such that

$$
F(k, \xi) \leq \varepsilon|\xi|^{p}+h_{\varepsilon}
$$

for each $\xi \in \mathbf{R}$ and $k \in \mathbf{Z}(1, T)$. According to Lemma 2.3 and due to $\lambda<\frac{m(2 c)^{p}}{F^{c} p(T+1)^{p-1}}$, one has

$$
\begin{aligned}
\lambda \sum_{k=1}^{T} F(k, u(k)) & \leq \lambda \sum_{k=1}^{T}\left[\varepsilon|u(k)|^{p}+h_{\varepsilon}\right] \\
& \leq \frac{\lambda \varepsilon T(T+1)^{p-1}}{2^{p}}\|u\|^{p}+\lambda T h_{\varepsilon} \\
& <\frac{\varepsilon T m c^{p}}{F^{c} p}\|u\|^{p}+\frac{m(2 c)^{p} T}{F^{c} p(T+1)^{p-1}} h_{\varepsilon}
\end{aligned}
$$

for each $u \in X$. Therefore, we have

$$
\begin{aligned}
\Phi(u)-\lambda \Psi(u) & =\frac{1}{p} \sum_{k=1}^{T+1} p(k)|\Delta u(k-1)|^{p}-\lambda \sum_{k=1}^{T} F(k, u(k)) \\
& >\frac{m}{p}\|u\|^{p}-\frac{\varepsilon T m c^{p}}{F^{c} p}\|u\|^{p}-\frac{m(2 c)^{p} T}{F^{c} p(T+1)^{p-1}} h_{\varepsilon} \\
& =\left[\frac{m}{p}-\frac{\varepsilon T m c^{p}}{F^{c} p}\right]\|u\|^{p}-\frac{m(2 c)^{p} T}{F^{c} p(T+1)^{p-1}} h_{\varepsilon}
\end{aligned}
$$

for $\|u\| \geq 1$. This gives $\lim _{\|u\| \rightarrow+\infty} \Phi(u)-\lambda \Psi(u)=+\infty$.

On the other hand, if

$$
\limsup _{|\xi| \rightarrow+\infty} \frac{F(k, \xi)}{|\xi|^{p}}<0
$$


there is a positive constant $h_{\varepsilon}$ such that $F(k, \xi) \leq h_{\varepsilon}$, and so arguing as before we have

$$
\begin{aligned}
\Phi(u)-\lambda \Psi(u) & =\frac{1}{p} \sum_{k=1}^{T+1} p(k)|\Delta u(k-1)|^{p}-\lambda \sum_{k=1}^{T} F(k, u(k)) \\
& >\frac{m}{p}\|u\|^{p}-\frac{m(2 c)^{p} T}{F^{c} p(T+1)^{p-1}} h_{\varepsilon}
\end{aligned}
$$

for $\|u\| \geq 1$. Again, we obtain $\lim _{\|u\| \rightarrow+\infty} \Phi(u)-\lambda \Psi(u)=+\infty$, and thereby condition $\left(a_{2}\right)$ holds.

In summary, all the hypotheses in Lemma 2.2 have been demonstrated to be true. Therefore, the functional $\Phi(u)-\lambda \Psi(u)$ has at least three different critical points for each $\lambda \in \Lambda_{r}$, and the proof is completed.

Now, let

$$
F^{+}(k, \xi)=\int_{0}^{\xi} f\left(k, t^{+}\right) d t, \quad(k, \xi) \in \mathbf{Z}(1, T) \times \mathbf{R},
$$

where $t^{+}=\max \{0, t\}$ and $I_{\lambda}^{+}=\Phi-\lambda \Psi^{+}$. Here $\Phi$ is defined by (2.1) and

$$
\Psi^{+}(u):=\sum_{k=1}^{T} F^{+}(k, u(k))
$$

Clearly, $I_{\lambda}^{+} \in C^{1}(X, \mathbf{R})$ and the critical points of $I_{\lambda}^{+}$provide the solutions to the following problem:

$$
\left\{\begin{array}{l}
\triangle\left[p(k) \phi_{p}(\triangle u(k-1))\right]+\lambda f\left(k, u^{+}(k)\right)=0, \quad k \in \mathbf{Z}(1, T) \\
u(0)=u(T+1)=0 .
\end{array}\right.
$$

We have the following

Corollary 3.2 Suppose that $c$ and $d$ are positive constants such that

$$
\frac{1}{p}|d|^{p}(p(1)+p(T+1))>\frac{m(2 c)^{p}}{p(T+1)^{p-1}},
$$

and satisfying

(i) $f(k, \xi)>0$ for each $k \in Z(1, T)$ and $\xi \in[0, d]$;

(ii) $\frac{p F^{d}}{|d|^{p}(p(1)+p(T+1))}>\frac{F^{c} p(T+1)^{p-1}}{m(2 c)^{p}}$;

(iii) $\limsup _{\xi \rightarrow+\infty} \frac{F(k, \xi)}{\xi^{p}}<\frac{F^{c}}{T c^{p}}$.

Then,for each $\lambda \in \Lambda_{r}$, problem (1.1) has at least three positive solutions.

Proof For any $k \in \mathbf{Z}(1, T)$, (3.14) should be considered with

$$
f^{+}(k, \xi)= \begin{cases}f(k, \xi), & \text { if } \xi>0 \\ f(k, 0), & \text { if } \xi \leq 0\end{cases}
$$

Under Theorem 3.2, one has that (i) is tenable. 
Moreover, we have

$$
\limsup _{\xi \rightarrow-\infty} \frac{F^{+}(k, \xi)}{|\xi|^{p}}=\limsup _{\xi \rightarrow-\infty} \frac{\xi f(k, 0)}{|\xi|^{p}}=-\limsup _{\xi \rightarrow-\infty} \frac{f(k, 0)}{|\xi|^{p-1}}=0<\frac{F^{c}}{T c^{p}}
$$

Consequently, all the requirements of Theorem 3.2 have been met. Besides, since $u \equiv 0$ is not a solution to (3.14), it can be inferred that (3.14) has at least three nontrivial solutions. Suppose that $u=\{u(k)\}$ is a nontrivial solution. Then for any $k \in \mathbf{Z}(1, T)$, we have $u(k)>0$ or

$$
-\triangle\left[p(k) \phi_{p}(\triangle u(k-1))\right]=\lambda f\left(k, u^{+}(k)\right)=\lambda f(k, 0)>0 .
$$

Therefore, according to Lemma 2.4, we may refer to $u(k)>0$ for $k \in \mathbf{Z}(1, T)$. This $u$ denotes a positive solution. In addition, once $u$ has been verified as a positive solution to (3.14), it can be also considered as a positive solution of (1.1). Thus, Corollary 3.2 is demonstrated.

\section{Examples}

We show three examples to confirm our findings in this section.

Example 4.1 Let $\alpha: \mathbf{Z}(1,2) \rightarrow \mathbf{R}$ be positive and let $y:[0,+\infty) \rightarrow \mathbf{R}$ be a function defined by

$$
y(u)= \begin{cases}u^{8}, & 0 \leq u<2, \\ (4-u)^{8}, & 2 \leq u<3, \\ 1, & u \geq 3 .\end{cases}
$$

Let $p=3, c_{1}=1, c_{2}=8, d=2, T=2, m=8, p(1)=1$, and $p(3)=2$. Then

$$
\frac{2 c_{1} m^{\frac{1}{P}}}{(T+1)^{\frac{p-1}{p}}(p(1)+p(T+1))^{\frac{1}{p}}}=\frac{2 \times 1 \times 8^{\frac{1}{3}}}{(3)^{\frac{2}{3}}(3)^{\frac{1}{3}}} \approx 1.333,
$$

and

$$
\frac{2 c_{2} m^{\frac{1}{P}}}{(T+1)^{\frac{p-1}{p}}(2(p(1)+p(T+1)))^{\frac{1}{p}}}=\frac{2 \times 8 \times 8^{\frac{1}{3}}}{(3)^{\frac{2}{3}}(2 \times(1+2))^{\frac{1}{3}}} \approx 8.466 .
$$

Thus, (3.1) holds. Since

$$
\frac{\int_{0}^{1} y(\xi) d \xi}{1^{3}} \approx 0.111, \frac{2 \int_{0}^{8} y(\xi) d \xi}{8^{3}} \approx 0.464
$$

and

$$
\frac{8 \times 2^{2} \int_{0}^{2} y(\xi) d \xi}{3^{3} \times 2^{3}} \approx 8.428
$$


we obtain

$$
\max \left\{\frac{\int_{0}^{1} y(\xi) d \xi}{1^{3}}, \frac{2 \int_{0}^{8} y(\xi) d \xi}{8^{3}}\right\}<\frac{8 \times 2^{2} \int_{0}^{2} y(\xi) d \xi}{3^{3} \times 2^{3}} .
$$

Applying Corollary 3.1, for each $\lambda \in(0.281 B, 5.109 B)$, where $B=1 / \sum_{k=1}^{2} \alpha(k)$, the following problem:

$$
\left\{\begin{array}{l}
\triangle\left[p(k) \phi_{3}(\Delta u(k-1))\right]+\lambda \alpha(k) y(u(k))=0, \quad k \in \mathbf{Z}(1,2), \\
u(0)=u(3)=0,
\end{array}\right.
$$

has at least two positive solutions.

Next, let us discuss another example.

Example 4.2 Consider the boundary value problem (1.1) with

$$
f(k, \xi)=f(\xi)=3+\sin \xi+\xi \cos \xi, \quad \xi \in[0,1]
$$

for $k \in \mathbf{Z}(1, T)$. It follows that

$$
F(k, \xi)=F(\xi)=\xi(3+\sin \xi) .
$$

Letting $c=1, d=2, p=2, p(1)=1, T=4, p(5)=2$, and $m=8$, one has

$$
\frac{1}{p}|d|^{p}(p(1)+p(5))=6>\frac{m(2 c)^{p}}{p 5^{p-1}}=3.2 .
$$

Obviously, $f(k, \xi)>0$ holds for each $\xi \in[0,1]$ and $k \in \mathbf{Z}(1, T)$. Accordingly, condition (i) in Corollary 3.2 can be obtained. Then, we have

$$
\frac{F^{c} p 5^{p-1}}{m(2 c)^{p}}=\frac{4(3+\sin 1) \times 10}{32} \approx 4.800
$$

and

$$
\frac{p F^{d}}{|d|^{p}(p(1)+p(5))}=\frac{4(6+2 \sin 2)}{6} \approx 5.212 .
$$

Therefore, combining (4.4) and (4.5), condition (ii) in Corollary 3.2 is verified.

We can show condition (iii) in Corollary 3.2, as

$$
\limsup _{\xi \rightarrow+\infty} \frac{F(k, \xi)}{\xi^{p}}=\limsup _{\xi \rightarrow+\infty} \frac{3+\sin \xi}{\xi^{p-1}}=0<\frac{F^{c}}{T c^{p}} .
$$

In summary, all the conditions in Corollary 3.2 have been checked. Therefore, for each $\lambda \in \Lambda_{r}=\left(\frac{6}{4(6+2 \sin 2)}, \frac{32}{40(3+\sin 1)}\right)$, problem (1.1) has at least three positive solutions.

Finally, let us discuss one more example. 
Example 4.3 Consider the special case of (1.1) with $p(k) \equiv 1$ and

$$
f(k, u)=f(u)=5+\sin u+u \cos u, \quad u \in[-1,2],
$$

for $k \in \mathbf{Z}(1, T)$. Therefore, we have

$$
F(k, u)=F(u)=u(5+\sin u)-5 .
$$

Letting $c=1, d=p=2, T=3, p(1)=p(4)=1$, and $m=1$, one has

$$
\frac{1}{p}|d|^{p}(p(1)+p(4))=4>\frac{m(2 c)^{p}}{p 4^{p-1}}=0.5 .
$$

We have $f(k, u)>0$ for every $u \in[-1,2]$. Thus, condition (i) in Theorem 3.2 is satisfied. We also have

$$
\frac{F^{c} p 4^{p-1}}{m(2 c)^{p}}=\frac{3 \sin 1 \times 8}{4} \approx 5.046
$$

and

$$
\frac{p F^{d}}{|d|^{p}(p(1)+p(4))}=\frac{3(5+2 \sin 2)}{4} \approx 5.115 .
$$

Therefore, according to (4.6) and (4.7), condition (ii) of Theorem 3.2 holds.

Noting that

$$
\limsup _{|u| \rightarrow+\infty} \frac{F(k, u)}{|u|^{p}}=\limsup _{|u| \rightarrow+\infty} \frac{u(5+\sin u)-5}{|u|^{p}}=0<\frac{F^{c}}{T c^{p}},
$$

condition (iii) in Theorem 3.2 can be further verified.

In summary, all the conditions of Theorem 3.2 have been verified. Then, for each $\lambda \epsilon$ $\Lambda_{r}=\left(\frac{4}{3(5+2 \sin 2)}, \frac{1}{6 \sin 1}\right)$, problem (1.1) has at least three nontrivial solutions.

We end this section by using the above hypothesis to compare our result with that of [36, Theorem 3.1]. Through careful calculations, we obtain

$$
\max _{(k, \xi) \in \mathbf{Z}(1, T) \times[-c, c]} F(k, \xi) \approx 0.841
$$

and

$$
\frac{(2 c)^{p}}{T\left[(2 c)^{p}+2(T+1)^{p-1} d^{p}\right]} \sum_{k=1}^{T} F(k, d) \approx 0.758 .
$$

Then it holds that

$$
\max _{(k, \xi) \in \mathbf{Z}(1, T) \times[-c, c]} F(k, \xi)>\frac{(2 c)^{p}}{T\left[(2 c)^{p}+2(T+1)^{p-1} d^{p}\right]} \sum_{k=1}^{T} F(k, d) .
$$


According to (4.8), it can be inferred that condition $\left(A_{1}\right)$ of [36, Theorem 3.1] is not satisfied. This shows that the conclusion about the three solutions using [36, Theorem 3.1] cannot be obtained.

\section{Acknowledgements}

We would like to thank the reviewers for their constructive and detailed comments, which greatly help us improve the presentation of this paper.

\section{Funding}

This work is supported by the National Natural Science Foundation of China (Grant No. 11971126), the Program for Changjiang Scholars and Innovative Research Team in University (Grant No. IRT_16R16) and the Innovation Research for the Postgraduates of Guangzhou University (Grant No. 2020GDJC-D07).

\section{Availability of data and materials}

Not applicable.

\section{Competing interests}

The authors declare that they have no competing interests.

\section{Authors' contributions}

All authors contributed equally to the writing of this paper. All authors read and approved the final manuscript.

\section{Publisher's Note}

Springer Nature remains neutral with regard to jurisdictional claims in published maps and institutional affiliations.

Received: 28 January 2021 Accepted: 22 March 2021 Published online: 01 April 2021

\section{References}

1. Elaydi, S.: An Introduction to Difference Equations, 3rd edn. Springer, New York (2005)

2. Long, Y.H., Wang, L.: Global dynamics of a delayed two-patch discrete SIR disease model. Commun. Nonlinear Sci. Numer. Simul. 83, 105117 (2020)

3. Yu, J.S., Zheng, B.: Modeling Wolbachia infection in mosquito population via discrete dynamical model. J. Differ. Equ. Appl. 25, 1549-1567 (2019)

4. Lin, G.H., Ji, J.P., Wang, L., Yu, J.S.: Multitype bistability and long transients in a delayed spruce budworm population model. J. Differ. Equ. 283, 263-289 (2021)

5. Lin, G.H., Yu, J.S., Zhou, Z., Sun, Q.W., Jiao, F.: Fluctuations of mRNA distributions in multiple pathway activated transcription. Discrete Contin. Dyn. Syst., Ser. B 24, 1543-1568 (2019)

6. Bohner, M., Hassan, T.S., Li, T.: Fite-Hille-Wintner-type oscillation criteria for second-order half-linear dynamic equations with deviating arguments. Indag. Math. 29, 548-560 (2018)

7. Bohner, M., Li, T.: Oscillation of second-order $p$-Laplace dynamic equations with a nonpositive neutral coefficient. Appl. Math. Lett. 37, 72-76 (2014)

8. Li, T., Pintus, N., Viglialoro, G.: Properties of solutions to porous medium problems with different sources and boundary conditions. Z. Angew. Math. Phys. 70, 1-18 (2019)

9. Henderson, J., Thompson, H.B.: Existence of multiple solutions for second order discrete boundary value problems. Comput. Math. Appl. 43, 1239-1248 (2002)

10. Bereanu, C., Mawhin, J.: Boundary value problems for second order nonlinear difference equations with discrete $\phi$-Laplacian and singular $\phi$. J. Differ. Equ. Appl. 14, 1099-1118 (2008)

11. Guo, Z.M., Yu, J.S.: The existence of periodic and subharmonic solutions for second order superlinear difference equations. Sci. China Ser. A 46, 506-515 (2003)

12. Zhou, Z., Ling, J.X.: Infinitely many positive solutions for a discrete two point nonlinear boundary value problem with $\phi_{c}$-Laplacian. Appl. Math. Lett. 91, 28-34 (2019)

13. Zhou, Z., Su, M.T.: Boundary value problems for 2 thth-order $\phi_{c}$-Laplacian difference equations containing both advance and retardation. Appl. Math. Lett. 41, 7-11 (2015)

14. Bonanno, G., Candito, P.: Infinitely many solutions for a class of discrete nonlinear boundary value problems. Appl. Anal. 88, 605-616 (2009)

15. D'Aguì, G., Mawhin, J., Sciammetta, A.: Positive solutions for a discrete two point nonlinear boundary value problem with p-Laplacian. J. Math. Anal. Appl. 447, 383-397 (2017)

16. Avery, R., Henderson, J.: Existence of three positive pseudo-symmetric solutions for a one dimensional discrete p-Laplacian. J. Differ. Equ. Appl. 10, 529-539 (2004)

17. He, Z:: On the existence of positive solutions of $p$-Laplacian difference equations. J. Comput. Appl. Math. 161, 193-201 (2003)

18. Li, Y., Lu, L.: Existence of positive solutions of $p$-Laplacian difference equations. Appl. Math. Lett. 19, 1019-1023 (2006)

19. Liu, Y., Ge, W.: Twin positive solutions of boundary value problems for finite difference equations with $p$-Laplacian operator. J. Math. Anal. Appl. 278, 551-561 (2003)

20. Shi, H.P.: Periodic and subharmonic solutions for second order nonlinear difference equations. J. Appl. Math. Comput. 48, 157-171 (2005)

21. Yu, J.S., Guo, Z.M., Zou, X.F.: Periodic solutions of second order self-adjoint difference equations. J. Lond. Math. Soc. (2) $71,146-160(2005)$ 
22. Encinas, A.M., Jimenez, M.J.: Bounded solutions of self-adjoint second order linear difference equations with periodic coefficients. Open Math. 16, 75-82 (2018)

23. Zhou, Z., Ma, D.F.: Multiplicity results of breathers for the discrete nonlinear Schrödinger equations with unbounded potentials. Sci. China Math. 58, 781-790 (2015)

24. Zhang, Q.Q.: Homoclinic orbits for discrete Hamiltonian systems with local super-quadratic conditions. Commun. Pure Appl. Anal. 18, 425-434 (2019)

25. Lin, G.H., Zhou, Z., Yu, J.S.: Ground state solutions of discrete asymptotically linear Schrödinger equations with bounded and non-periodic potentials. J. Dyn. Differ. Equ. 32, 527-555 (2020)

26. Zhou, Z., Yu, J.S., Chen, Y.M.: Homoclinic solutions in periodic difference equations with saturable nonlinearity. Sci. China Math. 54, 83-93 (2011)

27. Zhou, Z., Yu, J.S.: Homoclinic solutions in periodic nonlinear difference equations with superlinear nonlinearity. Acta Math. Sin. Engl. Ser. 29, 1809-1822 (2013)

28. Lin, G.H., Zhou, Z.: Homoclinic solutions of discrete $\phi$-Laplacian equations with mixed nonlinearities. Commun. Pure Appl. Anal. 17, 1723-1747 (2018)

29. Zhang, Q.Q.: Homoclinic orbits for a class of discrete periodic Hamiltonian systems. Proc. Am. Math. Soc. 143 3155-3163 (2015)

30. Zhang, Q.Q.: Homoclinic orbits for discrete Hamiltonian systems with indefinite linear part. Commun. Pure Appl. Anal. 14, 1929-1940 (2017)

31. Ma, M.J., Guo, Z.M.: Homoclinic orbits for second order self-adjoint difference equations. J. Math. Anal. Appl. 323, 513-521 (2006)

32. Lin, G.H., Yu, J.S., Zhou, Z:: Homoclinic solutions of discrete nonlinear Schrödinger equations with partially sublinear nonlinearities. Electron. J. Differ. Equ. 2019, 96 (2019)

33. Lin, G.H., Zhou, Z: Homoclinic solutions in non-periodic discrete $\phi$-Laplacian equations with mixed nonlinearities Appl. Math. Lett. 64, 15-20 (2017)

34. Ma, M.J.: Dominant and recessive solutions for second order self-adjoint linear difference equations. Appl. Math. Lett. 18, 179-185 (2005)

35. Long, Y.H., Yu, J.S., Guo, Z.M.: Disconjugacy and C-disfocality of second order self adjoint difference equation. Comput. Math. Appl. 49, 933-942 (2005)

36. Jiang, L., Zhou, Z:: Three solutions to Dirichlet boundary value problems for $p$-Laplacian difference equations. Adv. Differ. Equ. 2008, 345916 (2008)

37. Bonanno, G., Candito, P.: Non-differentiable functions with applications to elliptic equation with discontinuous nonlinearities. J. Differ. Equ. 244, 3031-3059 (2008)

38. Bonanno, G., Candito, P., D'Aguì, G.: Variational methods on finite dimensional Banach spaces and discrete problems. Adv. Nonlinear Stud. 14, 915-939 (2014)

\section{Submit your manuscript to a SpringerOpen ${ }^{\circ}$ journal and benefit from:}

- Convenient online submission

- Rigorous peer review

- Open access: articles freely available online

- High visibility within the field

Retaining the copyright to your article

Submit your next manuscript at $\gg$ springeropen.com 